\title{
KNOWLEDGE-CARRYING OBJECTS IN LEARNING SITUATIONS
}

\author{
Elin OLANDER ${ }^{1}$, Charlotte Asbjørn SÖRENSEN ${ }^{2}$, Enes MUSA ${ }^{2}$, Sara ANDERSSON $^{2}$ \\ and Marielle STERNER ${ }^{3}$ \\ ${ }^{1}$ Department of Design Science, Industrial Design, Lund University, Lund, Sweden \\ ${ }^{2}$ School of Art and Communication (K3), Malmö University, Malmö, Sweden \\ ${ }^{3} \mathrm{Cool}$ Minds, Science Centre for Children, Malmö, Sweden
}

\begin{abstract}
This study aims to explore the scope of 'knowledge-carrying' objects in learning situations. The study is based on a qualitative content analysis of a design project conducted in a science centre for children aged 7-10 years, learning about polymers and circularity. The design project is based on research through design and has been an iterative process where design experiments and observations were intertwined. The main purpose of the study has been to understand the role a 'knowledge-carrying' object may play in learning situations, and whether an object can convey knowledge between learners or between the object and the individual, without the presence of a facilitator. The study also aimed to explore what type of properties or requirements can be claimed by such an object. Different types of documentation from the design project has been analysed through a qualitative content analysis in a topdown process in order to explore and identify how the iterations of the objects changed the objects' capacity to carry knowledge during the project's time frame. The goal was to create an initial concept of how designers can work with 'knowledge-carrying' objects in learning situations. Finally, the understanding of the role of 'knowledge-carrying' objects is discussed in relation to other types of mediating objects used by designers in design processes and using 'knowledge-carrying' objects as pedagogical agents in design education.
\end{abstract}

Keywords: Research trough design, playification, knowledge-carrying objects, mediating objects, experiential learning

\section{INTRODUCTION}

Objects are central for product designers both as a tool for communication and as a tool for visualisation of ideas or statements. Hence, in a design process objects can be both the means and the goal. This is in alignment with Elsen's [1] proposal; to be able to design something involving cooperative work, there is a need to link individual thoughts to external representations. This can be resolved with mediating objects [1], here referring to the different tools used in a design process to visualise problems and solutions, such as a pen, the computer, prototypes etc. Further, in artistic research, the designed object is often regarded as a knowledge contribution to the discipline. So, if we consider an object to be a physical representation of the designers' idea, vision, thoughts, or in this case knowledge, that can be communicated to another person; how can we then make sure that the receiver understand the designer's intended message? Can an object carry knowledge to be interpreted or understood by someone else, other than the creator? Furthermore, can such 'knowledge-carrying' objects be used as a pedagogical agent in learning situations? If so, what type of properties should a 'knowledge-carrying' object possess? With these questions in mind we started to investigate the role of objects in a specific learning situation, a research through design-based project aiming to create an object which could teach young children about polymers and circular production. However, this paper will not present the design project as such but will instead focus on analysing the 'knowledge-carrying' objects and the interaction that took place in the project. Hence, the paper presents a qualitative content analysis of the process documentation from the design project named: The Plastic Transformer - an object with purpose to teach children about plastics, environmental impact and circularity. The design project is situated in a local science centre for children, age 5-15 years. The main task for the design project was to explore how to educate children 7-10 years about polymers and circular production. All activities taking place (design experiments, 
observations and design work) in the project was documented in either written form, audio recording, sketches or photos. The qualitative content analysis of the project's process documentation, was chosen in order to gain a deeper understanding of the following (a) what role a 'knowledge-carrying' object can play in a learning situation (b) if a 'knowledge-carrying' object can convey knowledge between learners or between the object and the individual learner, and (c) what properties or requirements can be claimed by such an object.

\section{MEDIATING OBJECTS}

In design practice, objects are often used when exploring user needs, e.g. in participatory workshops or focus groups interviews, where the designer or facilitator is moderating the session. These objects are sometimes referred to in literature as mediating objects. They might be a representation of a future design solution (e.g. 3D prototype) or a future scenario (e.g. mood boards, pictures) and are used to trigger the users' feedback on a specific design solution or problem. Hence, a mediating object both steer the users' conversation as well as give inspiration and facilitate their ability to imagine. However, this study was triggered by the opportunity to explore whether the general usage might be developed. Hence, can an object convey knowledge through interaction with a user, regardless of the presence of a moderator? If so, here lays an opportunity for expanding designers' capability to interact with users. The concept of mediating objects is not only used within the design discipline. It is also used in natural science such as explaining phenomenon in biology [2], diagrams in contemporary mathematics [3], or helping patients to heal in psychical therapeutic care [4]. Even though the concept of mediating object is given different usage, roles and properties within the different research subjects, there are commonalities. Some argue that the mediating object's main purpose is to convey knowledge [3] [2], whereas others present the mediating object as a tool for gaining new perspective on the individual's personal thoughts [4] [5]. Some claim that the physical object itself does not possess specific characteristics, it is an ordinary object like a photo, a doll or peas [2] [4]. That it is the purpose of usage, which transform the ordinary object into a mediating object. For others the mediating object acts as a third person in a dialogue, offering the user the possibility to deposit, project, recollect, receive, and transform sensorial perceptions and thoughts without any risk [4]. Hence, the object has the capacity to receive different imageries. This agrees with yet another explanation of a mediating object; that it has a greater capacity than other objects to mobilise, unify, and concentrate knowledge [5]. However, to do so, the mediating object should then represent the core of a common problem to be solved, to bring the users to come together, to reach a common understanding [5]. The object will thereby give the users the opportunity to confront old viewpoints and build new on the core topic to be explored. Additionally, the object also will reveal the user's attachment and commitment to contribute to solve the problem [5]. Another argument is that a mediating object is connected to the facts it is representing, hence playing the role of teaching the user [3]. The most important aspect here is that the users, by adding and removing details, without changing the given relationships between the presented facts, can manipulate the object. Thereby presenting knowledge becomes the main role of a mediating object, however how the presentation is done, affect the user's ability to understand and gain the knowledge [3]. This argument is in line with what this study would like to obtain with the 'knowledge-carrying' object. Hence the object becomes a mediator that teach the user by offering controlled manipulations. This require the user to be active to gain the knowledge that the object represent, hence the information should interrelate to the user's specific manipulations. Additionally, a mediating object cannot be understood independent of audience [2]. So, if a mediating object plays the role of teaching, the creator of the mediating object [3] need to be aware of that different audience will learn different things from the same representation. This would be in alignment with what the study aim to obtain when delving deeper into the process documentation of the design project, exploring the role and characteristics of a 'knowledge-carrying' object and the relation between the object and the user. Hence, the main categories: role, characteristics and relation, was chosen in the qualitative content analysis.

\section{EXPERIENTIAL LEARNING \& MEANINGFUL PLAY}

Experiential Learning Theory (ELT) or 'learning-by-doing' [6] is a holistic model that proposes a constructivist theory of learning, whereby social knowledge is created and recreated, within the personal knowledge of the learner [7]. 'Learning is the process whereby knowledge is created through the transformation of experience' [8]. The learning environment influences what role the learner plays in the learning activities and further mirrors the didactic design and reflects what role the learner is 
expected to play [9] [10]. By introducing a 'knowledge-carrying' object as the centre, or a hub of the learning environment, the object thereby contributes to creating a frame and a focus for the learning activity. Different aspects of the 'knowledge-carrying' object in relation to learning modes was explored through design experiments in the design project. In the last design experiment, carried out in the project, meaningful play or playification was used to encourage the learner to interact with the object, hence, to make it more engaging, enjoyable, and motivational. Playification begins by using the imagination to create narratives and stories that can frame the space around the user and includes rules, challenges, games, and gamification [11]. In order for play to be truly meaningful, one must create a free space in which one can play and explore in an environment where one can feel safe to try new things and fail, an environment in which ideas and objects can be shared [12]. These requirements are in line with the objectives of the study of a 'knowledge-carrying' object and how a mediating object is regarded or used. Playification as such is a method used to permeate an activity, task, or object with a psychological, emotional, social, or physical reward, and then evaluate the levels of extrinsic and intrinsic motivational forces. A playcentric approach requires continually keeping the users experience in mind, and continual evaluation and testing of the interaction of the user and object through every phase of design and development [13].

\section{DESIGN EXPERIMENTS - THE PLASTIC TRANSFORMER}

As mentioned above the playcentric approach was the main reason for the design project working with design experiments and observations intertwined and why it was carried out among the children visiting the local science centre. The design experiments were designed to explore different ways of how an object may disseminate knowledge but also to explore how the object interacted with both the environment it was placed in and the users. The result from an observation of the children's responses to the object and the placement of the object, influenced how the following design experiment were altered (Figure 1).

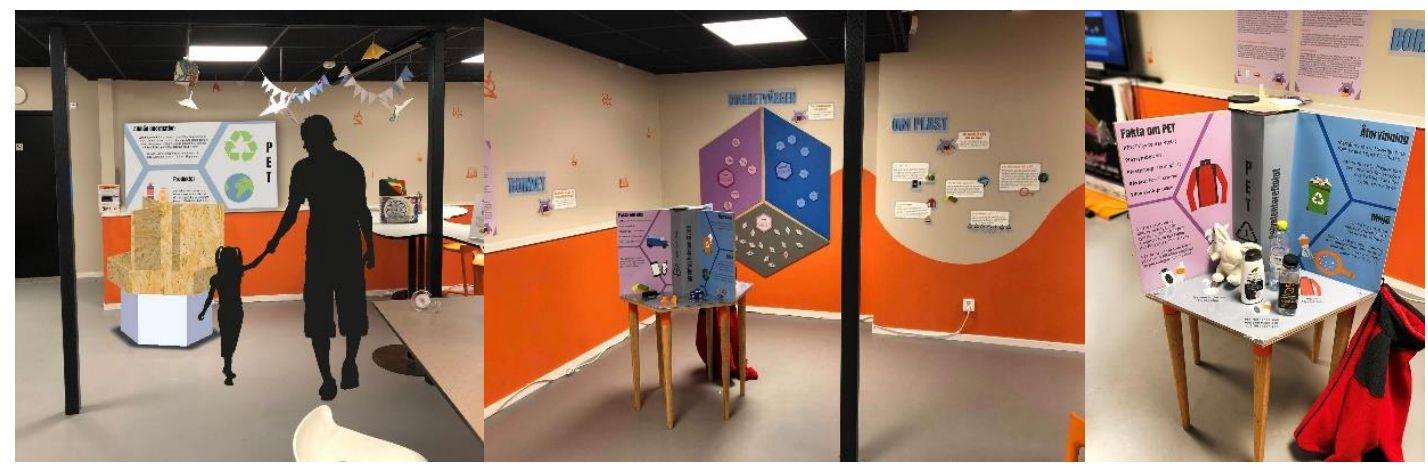

Figure 1. One of the design experiments from the Plastic Transformer project, exploring how a knowledge-carrying object interact with users and the surroundings

In the last design experiment playification was introduced as inspiration for the final development of the object. The final version of the design solution can be described as a playground, in the shape of a living space for a small character named the Plastic Transformer, which together with its friends and the children explore the material plastic, the materials usage and the materials ecological consequences and how it can be recycled (Figure 2). The design project formulated the following three hypotheses: (a) learning by interaction or information, (b) knowledge differentiation concerning age and pre-knowledge, and (c) play or tell (the object itself acting as a teacher or a best friend, where the last one is in alinement with the playification method). These three hypotheses were manifested in the objects used in the design experiments. All hypotheses have been derived from earlier insights on how children learn through learning-by-doing and meaningful play and in this case been exemplified through information about plastics and circularity. 


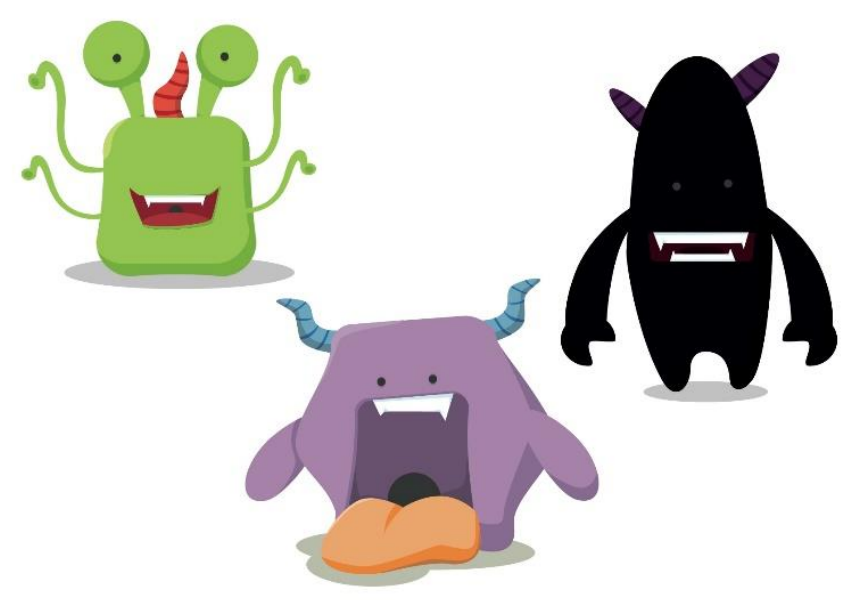

Figure 2. Personas used to explore the hypothesis "play or tell". The Plastic Transformer (purple) and the Recycler (green) teaches the Plastic Monster (black) how to act environmentally friendly

\section{QUALITATIVE CONTENT ANALYSIS}

The process documentation from the design project has been analysed through a qualitative content analysis [14]. The collected data constitutes a mix of notes, drawings, sketches and physical models, created and collected during the design project by the designers themselves. All activities in the design project, such as meetings, explorations, observations, brainstorming or prototyping has been documented by either written notes, audio recording, sketches or photos. Audio recordings has been transcribed, and some of the written notes has been transformed into visual representations. The qualitative content analysis has been done top-down with the main purpose to gain a deeper understanding of the 'knowledge-carrying' object's role in a learning situation, to identify common properties (if they exists), and further to capture the relation between object and user. The data from the design project was collected during a 4-month period. Therefore, the study also investigated if the understanding of the 'knowledge-carrying' object changed over time. The analysis has been carried out in the following steps:

1. the material was coded in three main categories: characteristics, role and relationship

2. within each of the three categories clusters were then identified and named

3. each cluster's content was then organized in individual timelines

4. each timeline was then analysed to identify if the meaning had changed or not over time

\section{FINDINGS}

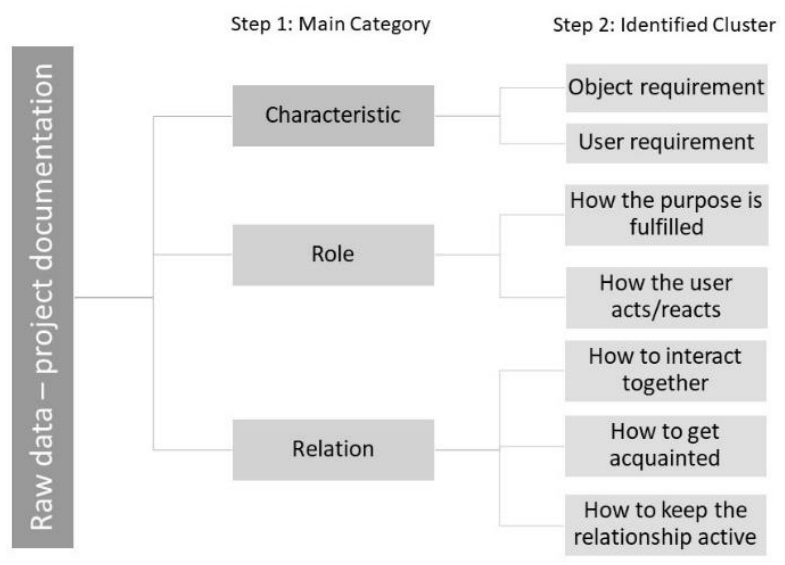

Figure 3. Overview of the content coding 
In the main category Characteristics, the content can be connected to either an object requirement or a user requirement (Figure 3). The content identified as user requirement indicate an active user whereas the content identified as object requirements indicate how the object behaves. In the category Role the content can be connected to either how the purpose is fulfilled or how the user acts/reacts on the purpose. One example; the purpose (to teach) can be fulfilled by sharing experience and the user's reaction can be to stretch given rules (given by the object) to change learning mode (self-motivational), and hence explore on their own. Finally, in the category Relation, the content can be connected to three different clusters: rules for how to interact (object and learner), how to get acquainted (introduce the learner to the object), and how to keep the relationship active (maintaining the learners' interest for interacting with the object). An example of how a rule can be obtained, could be that the objects' content can be adjusted based on the users pre-understanding or development. However, what was found most interesting, is that the content in the different clusters changed over time. Hence, the content is gradually changing from one interpretation to another. The fact that the change occur is not that surprising, since the 'knowledge-carrying' object is being explored through a design process and therefore the documentation used in the analysis is representing a process of change. What is interesting is how the change manifest itself. Why the change occur is more difficult to explain, one interpretation could be that the change is a result of the nature of an iterative design process, continuously shifting perspectives. This can be illustrated by how the focus initially, in the cluster titled object requirements represents the object's capability to present given information to the learner, and simultaneously support and adjust to the individual's needs. Whereas over time meaning of the content in the object requirements cluster changes from being an object to become an agent. Another example is in the cluster titled how the purpose is fulfilled where the change transforms from the object having a clear pedagogical purpose, acting as a teacher, mediating information and to become a personified object inviting to dialogue, playful interaction and thereby playful learning. In the cluster titled how the user acts/reacts there is a transformation from expecting the learner to leave evidence of achieved understanding towards signs of learning [15] and to have fun with their new knowledge-friends. However, in the cluster titled how to keep the relationship active, the project started with a distinct idea that a relationship is built upon safety and trust, growing stronger by continuously feedback. In the end it was the play itself that established the feeling of security and thereby created a balance and a shift of power, hence the object moved from being a teacher to become a playmate.

\section{DISCUSSION}

So, what role can a 'knowledge-carrying' object play in a learning situation? The content analysis indicated that the most important role for a 'knowledge-carrying' object is to provide a safe space for the learner to freely explore and play around with given content. This can be done by creating a sense of coherence and belonging between the object and the learner and create a mutual balance between give and take. To make the space truly safe and creating a meaningful play through playification can be one way to achieve that goal. During the study it also became clear that the role of the "knowledgecarrying' object is to bring people together to gain new knowledge through dialogue, to develop their prior understanding and by reflecting upon the knowledge the object represent. Thereby, the object and the user by 'playing' together can contribute to expose different perspective and point of views. A 'knowledge-carrying' object can convey knowledge between learners or between the object and the learner. This is the same requirements as placed on a mediating object. However, the 'knowledgecarrying' object as a designed object with an explicit purpose, in contrast to the claim that a mediating object can be any given object that has been given a specific role in a dialogue [2],[4]. The same way as a learning environment influences the learners' ability to learn [16], so does also the 'knowledgecarrying' object. The study indicates that a 'knowledge-carrying' object needs a framing. However, that framing is not required to be static or connected to a specific context, but the object must be adjusted to a specific audience. Therefore, should the 'knowledge-carrying' object be created with a given target group in mind. Hence, the starting point of creating a 'knowledge-carrying' object is to understand whom the learner is. Finally, so what properties or requirements can be claimed by a 'knowledgecarrying' object? The qualitative content analysis strongly indicates that a 'knowledge-carrying' object must encourage and invite the learner to interact with the object. When an interaction is established it must claim to be the safe space where the learner freely can modify and manipulate the object without destroying the interrelationship with the given content. The most important aspect when designing a 
'knowledge-carrying' object is to facilitate interaction. The analysis showed no common product properties that can be used to identify a 'knowledge-carrying' object. The study concludes that a 'knowledge-carrying' object can convey knowledge without a facilitator as a contrast to a mediating object. The challenge lies in creating an object that invites the learner to interaction. Further research is required to validate the findings and develop the concept of 'knowledge-carrying' objects and how to utilize it in design education.

\section{REFERENCES}

[1] Elsen C. Dawans A. and Leclercq P. An Anthropo-Based Study of Industrial Design Cooperative Practices Using "Mediating Objects". In $7^{\text {th }}$ international conference Cooperative Design, Visualization \& Engineering, CDVE 2010, Calvia, Mallorca, September 2010, 123-132.

[2] Ludwig D. Mediating Objects: Scientific and Public Functions of Models in Nineteenth-Century Biology. History and Philosophy of the Life Sciences, 2013, 35(2), 139-166.

[3] Carter J. Graph-algebras - Faithful representations and mediating objects in mathematics. Endeavour, 2018, 42(2-3), 180-188.

[4] Vacheret C. and Joubert C. Thinking about Synergy between the Group and the Mediating Object. Group Analysis, 2008, 41(3), 265-277.

[5] Steyaert P. The Maraîchine breed: a biological object mediating various forms of knowledge. International Social Science Journal, 2006, 58(187), 87-96.

[6] Dewey J. Experience and education for the 60th anniversary edition, 1938 (KAPPA DELTA PI).

[7] Kolb A. Y. and Kolb D. A. Learning styles and learning spaces: Enhancing experiential learning in higher education. Academy of Management Learning \& Education, 2005, 4(2), 193-212.

[8] Kolb D. A. Experiential learning. Experience as the source of learning and development, 1984 (Englewood Cliffs, N.J. Prentice-Hall cop).

[9] Dewey J. The school and society: Being three lectures by John Dewey supplemented by a statement of the university elementary school Chicago, 1907 (University of Chicago Press).

[10] Leijon M. Space as designs for and in learning: Investigating the interplay between space, interaction and learning sequences in higher education. Visual Communication, 2016, 15(1), 93124.

[11] Scott A. Meaningful play: how play is changing the future of our health, IDSA Education Symposium 2012, Boston. [Accessed on 2020, 8 January]

[12] Scott A. Meaningful play: how playcentric research methods are contributing to new understanding and opportunities for design. The Routledge companion to design research 2014 , pp.416-430.

[13] Fullerton T. Game design workshop a playcentric approach to creating innovative games, 2nd ed, 2008 (Morgan Kaufmann Publishers, Amsterdam).

[14] Williamson K. Given L. and Scifleet P. Qualitative data analysis. Research Methods: Information, Systems, and Contexts, 2013, pp.417-439.

[15] Selander S. and Kress G. Design för lärande: ett multimodalt perspektiv. 2017 (Studentlitteratur AB. Lund).

[16] Kress G. Assessment in the perspective of a social semiotic theory of multimodal teaching and learning. Educational Assessment in the $21^{\text {st }}$ Century 2009, pp.19-41. 